\title{
Effectiveness and Safety of Combined Application of Three Modes of 2940-nm Erbium:YAG and 1064-nm Neodymium:YAG Lasers in Treatment of Meibomian Gland Dysfunction
}

\author{
Ngamjit Kasetsuwan ${ }^{1,2}$ \\ Lita Uthaithammarat' \\ Yonrawee Piyacomn (D' \\ Usanee Reinprayoon ${ }^{1,2}$ \\ Chayanon Chatchavalvanich ${ }^{3}$ \\ Ratchathorn Panchaprateep $\mathbb{D}^{3}$ \\ 'Department of Ophthalmology, Faculty \\ of Medicine, Chulalongkorn University \\ and King Chulalongkorn Memorial \\ Hospital, Bangkok, Thailand; ${ }^{2}$ Excellence \\ Center of Cornea and Limbal Stem Cell \\ Transplantation, Department of \\ Ophthalmology, King Chulalongkorn \\ Memorial Hospital, Faculty of Medicine, \\ Chulalongkorn University, Bangkok, \\ Thailand; ${ }^{3}$ Division of Dermatology, \\ Department of Medicine, Faculty of \\ Medicine, Chulalongkorn University, \\ Bangkok, Thailand
}

\begin{abstract}
Purpose: To study the effectiveness and safety of upper and lower eyelid treatment with combined application of three modes of 2940-nm erbium-doped yttrium aluminium garnet (Er:YAG) and 1064-nm neodymium-doped yttrium aluminium garnet (Nd:YAG) lasers in patients with baggy eyelids (formed by intraorbital fat herniation) who exhibited meibomian gland dysfunction (MGD).

Patients and Methods: In this prospective cohort study, patients with baggy eyelid who exhibited MGD received combined laser treatment at baseline, as well as at the 4-, 8-, and 12-week follow-ups. The primary endpoint was meibum quality score at 16- and 24-week follow-ups; secondary endpoints were ocular surface index scores, tear film lipid layer thicknesses, tear break up times (TBUTs), Oxford scheme grades, and meibography grades at 16- and 24-week follow-ups. Adverse events, uncorrected visual acuities, best-corrected visual acuities, and intraocular pressures were also recorded.

Results: Sixteen patients (four men and 12 women; mean age, $56.38 \pm 8.64$ years) were included. Meibum quality scores at the 16-week follow-up were significantly lower than scores at baseline $(\mathrm{p}=0.043)$ and at the 24 -week follow-up $(\mathrm{p}=0.015)$. TBUT was significantly exacerbated at the 24-week follow-up, compared with baseline $(p=0.001)$ and the 16 week follow-up $(p=0.006)$. There were no significant changes in other parameters. All adverse events were mild and resolved without additional treatment.

Conclusion: Combined application of three modes of 2940-nm Er:YAG and 1064-nm Nd: YAG lasers on upper and lower eyelids significantly improved meibum quality in patients with MGD; it ameliorated symptoms and signs of dry eye disease at 4 weeks after completion of laser treatment.
\end{abstract}

Keywords: dry eye, erbium:YAG, meibomian gland dysfunction, meibum, neodymium: YAG

\section{Introduction}

Meibomian gland dysfunction (MGD) is a chronic secretion abnormality involving lipid glands embedded in both upper and lower eyelids, characterized by duct obstruction and/or qualitative/quantitative changes in meibum secretion. Lipid secretion from meibomian glands (MGs) is a main component of tear film, which plays an important role in preventing tear film evaporation; dysfunction involving these glands is a leading cause of evaporative dry eye, despite normal tear
Correspondence: Ngamjit Kasetsuwan King Chulalongkorn Memorial Hospital, Thai Red Cross Society, 1873 Rama 4 Road, Pathumwan, Bangkok, 10330 , Thailand

Tel +662-256-4l42

Email Ngamjitk@gmail.com 
secretion. ${ }^{1}$ Symptoms of dry eye disease due to MGD vary from none to ocular discomfort, redness, itching, or photophobia. $^{2}$ Severe dry eye disease may eventually lead to corneal erosion, infection, and scarring, which cause gradual vision loss. ${ }^{3}$

The mainstay treatment of MGD mainly involves warm compression, lid massage, and lid hygiene to melt the meibum, reopen the obstructed gland, and remove the meibum, as well as the use of anti-inflammatory agents to reduce inflammation and improve meibum quality. ${ }^{2}$ However, these treatments require robust patient compliance. Currently, there are various device-based treatments including thermal pulsation, intense pulsed light, and quantum molecular resonance. ${ }^{4,5}$ However, these devices have some limitations; for example, the thermal pulsation device activator does not fit in patients with small palpebral fornixes (especially Asian individuals), intense pulsed light might not be effective in patients who have very dark brown to black skin. ${ }^{6}$ To our knowledge, there have been few studies ${ }^{5,7}$ regarding the effectiveness of quantum molecular resonance. Therefore, we have investigated potential novel minimally invasive devices for treatment of MGD.

Non-ablative thermal laser treatment provides thermal effects alone (eg, coagulation) and photochemical effects without tissue ablation; ${ }^{8}$ this treatment includes three different modes of 2940-nm erbium-doped yttrium aluminium garnet (Er:YAG) and 1064-nm neodymium-doped yttrium aluminium garnet (Nd:YAG) lasers, which are generally used in skin rejuvenation and facial tightening. ${ }^{9-11}$ These lasers distribute heat deep under the mucosa, thereby enhancing collagen synthesis and vascularisation. ${ }^{9-14}$ Eyelid temperature considerably influences the physical properties of meibomian gland secretions. At room temperature, the eyelid temperature is approximately $33^{\circ} \mathrm{C}$. At higher temperatures, meibum becomes less viscous (phase-transition temperature), which facilitates its typical distribution over the cornea surface. The phase-transition temperature of meibum is higher in patients with MGD than in patients with healthy MGs. ${ }^{15}$ The properties of Er:YAG and Nd:YAG lasers are presumed to facilitate enhancement of meibum secretion from MGs. Heat production over the skin epidermis is presumed to exceed the phase-transition temperatures of meibum and collagen remodelling in the dermis, leading to periocular skin tightening that enhances eye lid margin apposition and blink completion, thereby enhancing meibum secretion from MGs. ${ }^{16}$
In this prospective observational study, we investigated the effectiveness and safety of combined treatment with three modes of 2940-nm Er:YAG and 1064-nm Nd:YAG lasers in patients with baggy eyelids (formed by intraorbital fat herniation) who exhibited MGD. All patients received this intervention to alleviate baggy eyelids in accordance with a dermatologist's recommendation.

\section{Materials and Methods}

This single-group, prospective cohort study was approved by the Institutional Review Board of the Faculty of Medicine, Chulalongkorn University, Thailand; the study protocol adhered to the tenets of the Declaration of Helsinki. Written informed consent and demographic data were obtained from all patients enrolled in this study. Moreover, the patient in Figure 1 provided inform consent for these image to be published. This study was conducted at the outpatient clinic, King Chulalongkorn Memorial Hospital, from March 2019 to October 2019.

\section{Participants}

Eligible patients were $\geq 18$ years of age and had been diagnosed with baggy lower eyelid; they also had a clinical diagnosis of MGD (any stage, based on the International Workshop on MGD grading scale ${ }^{17}$ ) and tear break-up time (TBUT) $<10$ second (s). For each participant, the eye with higher meibum quality score was included in the study. Exclusion criteria were as follows: 1) presence of ocular surface inflammation; 2) contact lens wear within the prior 1 month and during the study period; 3) any use of anti-glaucomatous eye drops within the prior 3 months and during the study period; 4) recent ocular or eyelid surgery; 5) presence of neuro-paralysis in the planned treatment area; 6) refractive surgery within the prior 6 months; 7) diagnosis of diabetes; 8) diagnosis of any autoimmune disease; and 9) need for enhanced safety (ie, ongoing use of punctual plugs; pre-cancerous lesions, skin cancer, or pigmented lesions in the planned treatment area; delayed wound healing disorder and abnormal scarring at the laser treatment site; presence of disease in the planned treatment area that might be affected by laser application, such as herpes simplex 1 and 2 or lupus; uncontrolled infections or uncontrolled immunosuppressive diseases; use of photosensitive medications and/or herbs, such as isotretinoin or tetracycline; pregnancy and/or lactation; legal blindness in one eye). 


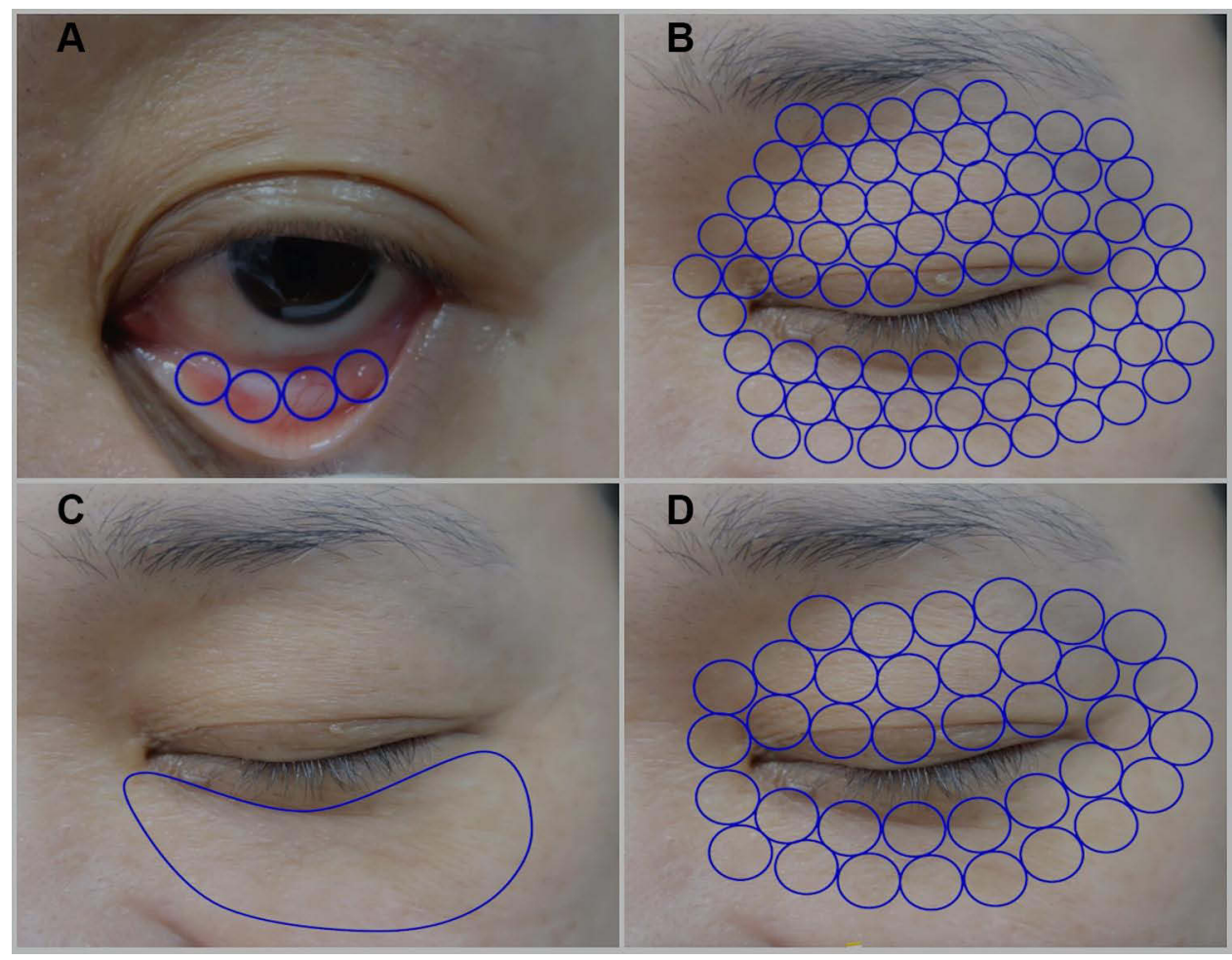

Figure I Treatment areas of steps I, 2, 3, and 4 of laser treatment. (A) Ten passes with 10 stag pulses using transconjunctival approach in step I. (B) Three passes with three stag pulses using cutaneous approach on periorbital skin of upper and lower eyelids in step 2 . (C) Five passes or until the skin temperature exceeds $40-42^{\circ} \mathrm{C}$ for $2-3$ minutes using cutaneous approach on bulging fat in the lower eyelid in step 3. (D) Three passes using cutaneous approach on periorbital skin of upper and lower eyelids in step 4.

\section{Treatment}

All patients with baggy eyelid problem was underwent treatment with Er:YAG and Nd:YAG lasers by Fotona SP Dynamis (Fotona, Ljubljana, Slovenia) using four-step laser modules-2940-nm Er:YAG SmoothLifting ${ }^{\text {TM }}$ (transconjunctival approach), 2940-nm Er:YAG SmoothLifting ${ }^{\text {TM }}$ (upper and lower lid), 1064-nm Nd:YAG PIANO ${ }^{\circledR}$ (lower lid), and 2940-nm Er:YAG SupErficial ${ }^{\mathrm{TM}}$ (upper and lower lid) — by the same surgeon investigator (R.P.) throughout the study. All patients received this intervention because of a baggy eyelid problem, in accordance with a dermatologist's recommendation. The investigators in this study did not initiate this treatment because of an MGD problem. Furthermore, this four-step laser was the treatment regimen for baggy eyelid. Before initiation of laser treatment, all patients received one eye drop of topical $0.5 \%$ tetracaine (Alcon Laboratories, Inc., Fort Worth, Texas) in each eye; oxytetracycline with polymyxin B sulfate ophthalmic ointment (Pfizer, Jakarta, Indonesia) was applied in both eyes immediately prior to the application of laser-impenetrable metal corneal shields. Each patient assumed the supine position, with the surgeon investigator sitting behind the patient's head. The surgeon investigator began with SmoothLiftin mode in the first step. This mode used a non-ablative Er:YAG laser, which was delivered in a unique $<1$-s pulse sequence with a repetition rate of $2 \mathrm{~Hz}$. The energy of each pulse ranged from 3.5 to $5 \mathrm{~J} / \mathrm{cm}^{2}$. In step 2 , the same mode was used as in step 1, but the repetition rate was changed to $3.3 \mathrm{~Hz}$. In step 3, the PIANO mode was used with a different handpiece (an R34 hand piece) and a spot size of $15 \mathrm{~mm}$. The PIANO mode, which incorporated a super-long Nd:YAG pulse, was a super-extended second regime mode with a brushing technique that provided heat throughout the dermis and into the subcutaneous tissue without damaging the epidermis. In this mode, pulse width (ie, time measured across a pulse based on the full width at a half-maximum of the pulse) was $16 \mathrm{~s}$. In the final step (SupErficial mode), light cold Er:YAG ablation was performed using the same PS03 handpiece that had been used in steps 1 and 2. The parameters of each laser module are shown in Table 1.

Laser treatments were performed at different sites for each step, as shown in Figure 1, at the first visit (baseline), 4-week follow-up, 8-week follow-up, and 12-week followup. Patients were instructed to use preservative-free artificial tears $(0.18 \%$ sodium hyaluronate) four times daily in 
Table I Sequences and Parameters of Four-Step Laser Treatment Applied to Upper and/or Lower Eyelids

\begin{tabular}{|c|c|}
\hline & Parameter \\
\hline $\begin{array}{l}\text { Step I: Er:YAG } \\
\text { SmoothLiftin }\end{array}$ & $\begin{array}{l}\text { - PS03 handpiece (Spot size } 5 \mathrm{~mm} \text { ) } \\
\text { - Smooth mode (duration } 250 \mathrm{msec} \text { ) } \\
\text { - Fluence } 3.5-5 \mathrm{~J} / \mathrm{cm} 2 \\
\text { - Freque } 3 \mathrm{ncy} 2 \mathrm{~Hz}\end{array}$ \\
\hline $\begin{array}{l}\text { Step 2: Er:YAG } \\
\text { SmoothLiftin }\end{array}$ & $\begin{array}{l}\text { - PS03 handpiece (Spot size } 5 \mathrm{~mm} \text { ) } \\
\text { - Smooth mode (duration } 250 \mathrm{msec} \text { ) } \\
\text { - Fluence } 3.5-5 \mathrm{~J} / \mathrm{cm} 2 \\
\text { - Frequency } 3.3 \mathrm{~Hz}\end{array}$ \\
\hline $\begin{array}{l}\text { Step 3: Nd:YAG } \\
\text { PIANO }^{\circledR}\end{array}$ & $\begin{array}{l}\text { - R34 handpiece (Spot size } 15 \mathrm{~mm}) \\
\text { - Sweep mode } \\
\text { - Fluence } 300-500 \mathrm{~J} / \mathrm{cm} 2 \\
\text { - Pulse width } 16 \mathrm{sec}\end{array}$ \\
\hline $\begin{array}{l}\text { Step 4: Er:YAG } \\
\text { SupErficial }^{T M}\end{array}$ & $\begin{array}{l}\text { - PS03 handpiece (Spot size } 7 \mathrm{~mm} \text { ) } \\
\text { - Micro-short pulse mode (duration } 100 \\
\text { microsec) } \\
\text { - Fluence } 2 \mathrm{~J} / \mathrm{cm} 2 \\
\text { - Frequency } 10 \mathrm{~Hz}\end{array}$ \\
\hline
\end{tabular}

Abbreviations: Er, erbium; YAG, yttrium aluminium garnet; Nd, Neodymium.

both eyes, throughout the study period. All other medications used to treat MGD (eg, oral antibiotics, supplements, and topical antibiotics or corticosteroids) were discontinued 2 weeks prior to the baseline examination.

\section{Outcome Measurement}

Demographic data were collected for all patients prior to the baseline examination. The primary endpoint of this study comprised meibum quality scores. The secondary endpoints were ocular surface disease index (OSDI) scores, tear film lipid layer thicknesses (TFLLTs), TBUTs, Oxford scheme grades, and meibography grades. OSDI scores were elicited from a 12-item questionnaire designed to provide rapid assessment of symptoms of ocular irritation (consistent with dry eye disease) and their impacts on vision-related functioning. ${ }^{18}$ To evaluate tear film stability, TBUT was measured in seconds (s) and evaluated three times per visit through the use of fluorescence strips and one drop of $0.9 \%$ normal saline. The average TBUT of three measurements was recorded. Oxford scheme grades were obtained under cobalt-blue light after fluorescein staining; staining severity was quantified using a chart with a series of panels. To grade the staining, its appearance on the exposed interpalpebral region of nasal and temporal conjunctiva and cornea was compared to the panel; keratoconjunctival staining severity was rated as stage $0,1,2,3,4$ and $5 .{ }^{19}$ Meibography comprises a specialised imaging analysis developed exclusively for direct visualisation of MG morphology in vivo. The Oculus Keratograph $5 \mathrm{M}^{\circledR}$ (Oculus, Wetzlar, Germany), a noncontact, placido ring-based corneal topography device, was used for meibography in this study. The degree of $M G$ dropout was graded as follows: Grade 0, no loss of MGs; Grade 1, 1-33\% loss of MG area; Grade 2, 33-67\% loss of MG area; and Grade 3, $>67 \%$ loss of MG area. ${ }^{20}$ Meibum quality was assessed after application of force to the eyelids with the Meibomian Gland Evaluator (TearScience Inc., Morrisville, NC, USA); the quality of expressed meibum (from eight glands in the central one-third of the lower eyelid) was graded as follows: Grade 0, normal and clear oil; Grade 1, cloudy meibum; Grade 2, cloudy meibum with debris; and Grade 3, thick or toothpaste-like meibum. ${ }^{20}$

Throughout the study, safety evaluations included any adverse events, as well as uncorrected visual acuity, bestcorrected visual acuity, and intraocular pressure. In addition, OSDI scores were recorded by a well-trained research assistant. TFLLT, TBUT, Oxford scheme grade, meibography grade, and meibum quality data were all recorded solely from the chosen eye of all patients at baseline, 16-week follow-up (4 weeks after completion of laser treatment), and 24-week follow-up (12 weeks after completion of laser treatment) by a single investigator (N.K.) throughout the study period.

\section{Statistical Analysis}

Demographics and baseline clinical characteristics are reported as means (standard deviations). Categorical data are shown as frequencies and percentages. Linear mixed model regression with a random intercept model was used for analyses of OSDI scores, uncorrected visual acuities, best-corrected visual acuities, TFLLTs, intraocular pressures, TBUTs, Oxford scheme grades, meibography grades, and meibum quality scores over time; the results are presented as estimated means and 95\% confidence intervals. To control type I error, Scheffe's method was used for multiple comparisons. All statistical tests were two-sided and used a significance threshold of $p<0.05$; Stata version 15.1 (StataCorp, College Station, TX, USA) was used for statistical analyses.

\section{Results}

Sixteen patients were enrolled in this study (four men and 12 women; mean age, $56.38 \pm 8.64$ years). One patient was lost to follow-up prior to the 24 -week visit. 
None of the patients had undergone prior refractive surgery or worn contact lenses. One patient had undergone lower eyelid blepharoplasty in both eyes, 1 year prior to participation in this study; one patient had received an eyelid line tattoo, 10 years prior to participation in this study. Other underlying diseases in the patients included hypertension in three patients, two of whom had dyslipidemia; two patients with hypertension used betablocker antihypertensive treatment, while one patient with hypertension was not using medication. Additionally, dyslipidemia was present in seven of the 16 patients; four used simvastatin, one used rosuvastatin, one used fenofibrate, and one did not use any medication. Estimated mean OSDI scores, TFLLTs, TBUTs, Oxford scheme grades, meibography grades, and meibum quality scores are shown in Table 2 and Figure 2.

\section{Primary Endpoint}

Meibum quality scores were significantly lower at the 16week follow-up than at baseline ( $\mathrm{p}=0.043)$ or the 24-week follow-up $(\mathrm{p}=0.015)$. However, there was no significant difference between meibum quality score at baseline and at the 24-week follow-up.

\section{Secondary Endpoints}

Pairwise comparison revealed no significant differences in OSDI scores, TFLLTs, Oxford scheme grades, or meibography grades among the three time points. The meibomian gland morphologies did not change between the first and last visits, as shown in Figure 3. Notably, TBUT was significantly worse at the 24-week follow-up than at baseline $(p=0.001)$ or the 16-week follow-up ( $\mathrm{p}=0.006)$. However, there was no significant difference between TBUT at baseline and at the 16week follow-up.

\section{Safety Assessments}

Some mild and temporary adverse events occurred in this study, all of which resolved spontaneously within 1 week without treatment. In 13 patients, mild conjunctival hyperemia was observed, as graded by the Cornea and Contact Lens Research Unit; ${ }^{21}$ this symptom resolved within 1 day of laser treatment. Notably, it occurred at one visit in some patients, and at two or three visits for other patients. Mild eyelid swelling immediately after laser treatment was observed in one patient after the second laser treatment; it disappeared within 2 days. Mild eye irritation during the second and third laser treatments was observed in two patients. Moreover, no changes in uncorrected visual acuity, best-corrected visual acuity, or intraocular pressure were observed during the study.

\section{Discussion}

This study assessed the effectiveness and safety of upper and lower eyelid treatment with combined application of three modes of 2940-nm Er:YAG and 1064-nm Nd:YAG lasers in patients with baggy eyelids who exhibited MGD. The primary endpoint, improvement of meibum quality, was achieved in this study. Patients also demonstrated mitigation of symptoms at the 16-week follow-up. In particular, mean OSDI scores indicated a change from moderate severity to mild severity at the 16-week followup. The Oxford scheme grade tended to decrease at the 16week follow-up, although this change was not statistically significant. However, TFLLTs and meibography grades did not significantly change after combination laser treatment.

There are many available treatments for MGD, including heat-based and non-heat-based methods. ${ }^{4}$ The phasetransition temperature of meibum is higher in patients with MGD $\left(35^{\circ} \mathrm{C}\right)$ than in patients with healthy MGs (32$\left.33^{\circ} \mathrm{C}\right) .{ }^{15}$ Moreover, the tarsal conjunctiva in patients with MGD $\quad\left(32.4-32.7^{\circ} \mathrm{C}\right)$ exhibit considerably lower

Table 2 Clinical Characteristics at Baseline and at 16-Week and 24-Week Follow-Ups

\begin{tabular}{|l|c|c|c|}
\hline \multirow{2}{*}{} & Baseline & I6-Week Follow-Up & 24-Week Follow-Up \\
\cline { 2 - 4 } & Mean [95\% CI] & Mean [95\% CI] & Mean [95\% CI] \\
\hline OSDI scores & $25.41[16.60,34.22]$ & $22.18[13.37,30.99]$ & $31.35[22.29,40.42]$ \\
TFLLT & $61.81[48.87,74.76]$ & $63.5[50.55,76.45]$ & $69.62[56.36,82.88]$ \\
TBUT(s) & $3.75[3.04,4.46]$ & $3.48[2.77,4.19]$ & $2.09[1.37,2.82]$ \\
Oxford scheme grade & $2.50[1.51,3.49]$ & $1.94[0.95,2.93]$ & $2.68[1.67,3.69]$ \\
Meibography grade & $1.50[1.16,1.84]$ & $1.38[1.04,1.7 I]$ & $1.26[0.92,1.61]$ \\
Meibum quality scores & $11.81[9.57,14.06]$ & $7.75[5.50,10.00]$ & $12.53[10.21,14.85]$ \\
\hline
\end{tabular}

Abbreviations: $\mathrm{Cl}$, confidence interval; OSDI, Ocular Surface Disease Index; TFLLT, tear film lipid layer thickness; TBUT, tear break-up time; s, seconds. 

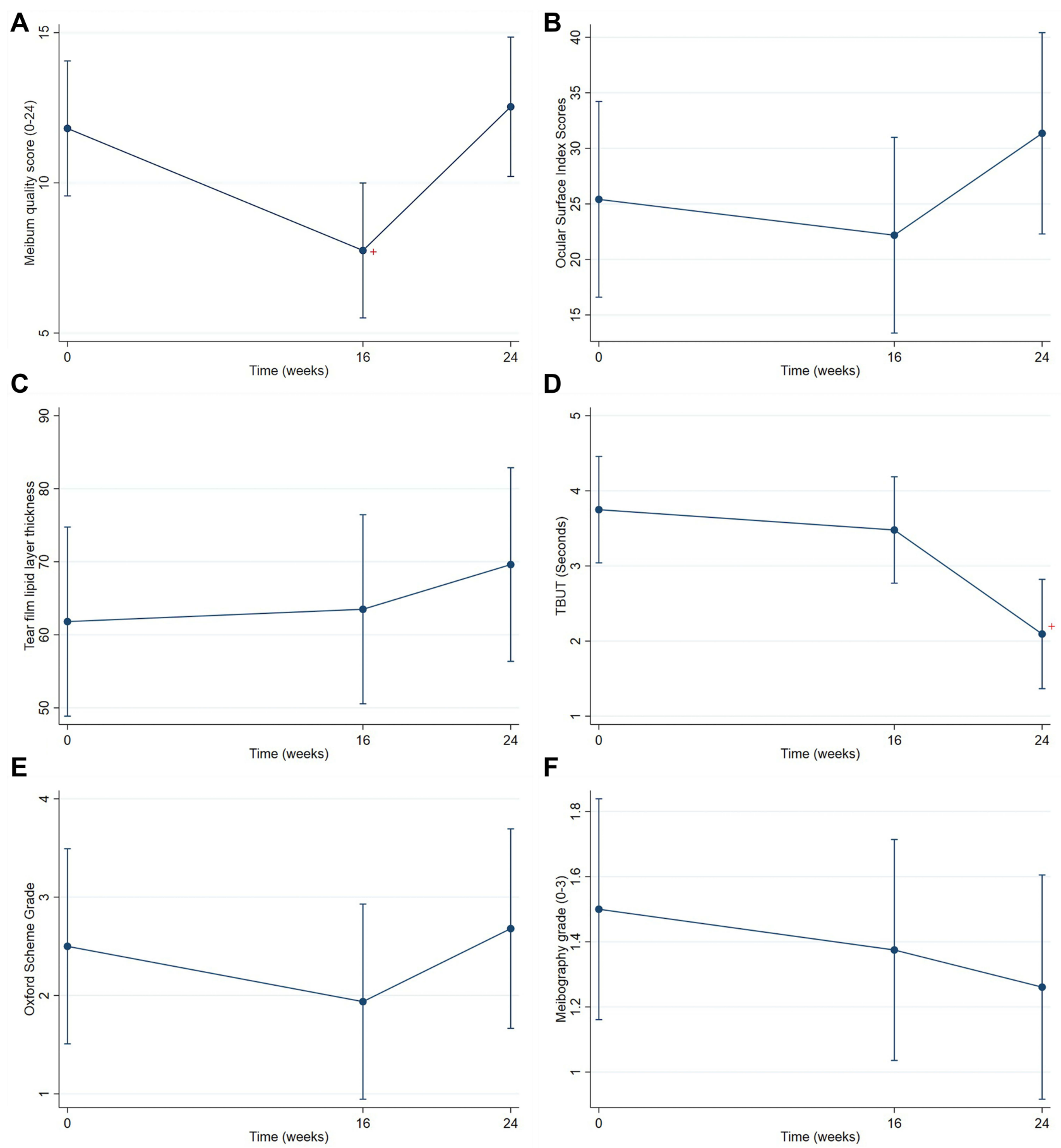

Figure 2 Estimated means with 95\% confidence intervals of clinical outcomes at each visit. (A) Estimated mean meibum quality scores; + represents significant difference compared with meibum quality scores at baseline and at 24 weeks. (B) Estimated mean Ocular Surface Disease Index scores. (C) Estimated mean tear film lipid layer thicknesses. (D) Estimated mean tear break-up times (TBUTs) in seconds; + represents significant difference compared with TBUT at baseline and at I6 weeks. (E) Estimated mean Oxford scheme grades. (F) Estimated mean meibography grades.

temperatures than the tarsal conjunctiva in healthy individuals $\left(34.3^{\circ} \mathrm{C}\right) .{ }^{22}$ Therefore, patients with MGD require heat to liquefy the thickened meibum. Recently, many studies reported the effectiveness of intense pulsed light in treatment of MGD; the presumed underlying mechanism was that the laser wavelength could reduce telangiectasia on the eyelids, thereby diminishing the inflammation involved in the onset of MGD. ${ }^{4}$ Moreover, laser photomodulation could stimulate mitochondria within MGs, which might improve MG microstructure and function. ${ }^{4}$ Importantly, Ferrari et $\mathrm{al}^{5}$ demonstrated the safety and efficacy of quantum molecular resonance- 


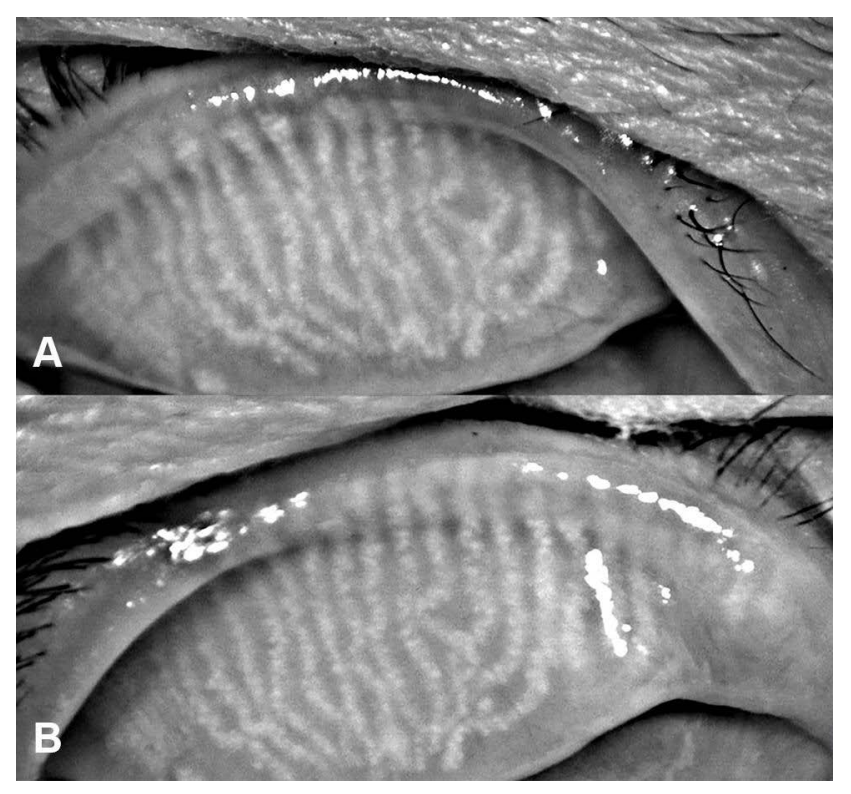

Figure 3 Meibomian gland morphology in a patient with meibomian gland dysfunction. (A) Meibomian gland morphology before treatment. (B) Meibomian gland morphology at 4 weeks after final treatment. Meibomian gland morphologies were similar in panels (A and $\mathbf{B})$.

based electrotherapy for treatment of MGD in a recent prospective study; the presumptive underlying mechanism involved anti-inflammatory effects. ${ }^{5}$ However, the optimal treatment for MGD has not been established.

Herein, we observed the effectiveness of combination application of three modes of 2940-nm Er:YAG and 1064$\mathrm{nm}$ Nd:YAG lasers, which produced higher eyelid temperature. This combination laser treatment significantly improved meibum quality in patients with MGD at 4 weeks after completion of treatment (ie, at the 16-week follow-up); however, the meibum quality had worsened by 12 weeks after completion of treatment (ie, at the 24-week follow-up). Similarly, as shown in Figure 2, OSDI scores and Oxford scheme grades tended to exhibit improvement at the 16-week follow-up, which was absent at the 24 week follow-up; however, this change at the 16-week follow-up was not statistically significant. These results indicated that the combined laser treatment was effective, but only for a short period of approximately 4 weeks after completion of treatment. Notably, there was no improvement in TBUT at the 16-week follow-up, whereas it was significantly exacerbated at the 24-week follow-up.

Using a cutaneous approach, the non-ablative 2940-nm Er: YAG laser has been shown to enable tissue heating of 45 $60^{\circ} \mathrm{C}$ at a depth of approximately $500 \mu \mathrm{m}$ in the upper dermis. $^{12}$ The heat from this approach may reach MGs because their acini near the mucocutaneous junction are approximately $417 \mu \mathrm{m}$ from the epithelial surface. ${ }^{23}$ Using a transconjunctival approach, the non-ablative 2940-nm Er: YAG laser directly heats the palpebral conjunctiva without causing corneal injury. This step could help increase the tarsal conjunctiva temperature in patients with MGD, such that it reaches a temperature closer to that of individuals with healthy MGs. ${ }^{22}$ The transmitted heat from the Er:YAG laser has been proposed to liquefy meibum in patients with MGD because the meibum temperature exceeds the phase-transition threshold. Moreover, heat from the Er:YAG laser could induce new collagen fibre generation, thereby improving the thickness and elasticity of eyelid skin., ${ }^{9,24,25}$ The improved eye lid skin exhibits better lid margin apposition and more complete blinks, which enhances meibum secretion from MGs. ${ }^{16}$ Furthermore, the Er:YAG laser was reported to significantly enhance levels of prostaglandin E2 (an angiogenesis factor) in human gingival fibroblasts. ${ }^{26}$ Prostaglandin E2 is presumably involved in fat synthesis within MGs. Both energy and oxygen via blood supply are required for fat synthesis. ${ }^{1}$ Notably, a positive relationship between lipocyte density and capillary density has been observed, such that lipocytes are more likely to cluster around large blood vessels. ${ }^{27}$ Consequently, we suspected that the Er:YAG laser might enhance prostaglandin E2 levels in human eyelid and contribute to the improvement of fat synthesis in MGs.

The added effect of the 1064-nm Nd:YAG laser was that it allowed laser energy to penetrate deeper into the dermis, which has been shown to affect collagen regeneration and remodelling in deep dermis. ${ }^{11,28}$ This mechanism was presumed to enhance eyelid elasticity, leading to greater meibum secretion. Moreover, during application of the 1064-nm Nd:YAG laser in our protocol, the skin temperature exceeded the range of $40-42^{\circ} \mathrm{C}$. Therefore, the eyelid temperature in our study exceeded the meibum phase transition threshold in patients with MGD during the Nd:YAG laser phase, which reduced the viscosity of thickened meibum in MGs of patients with MGD.

There were significant improvements of meibum quality scores at early follow-up, as well as tendencies for improvements of Oxford scheme grades and OSDI scores, although these tendencies were not statistically significant; however, TBUTs and meibography grades did not show improvement throughout the study. Many prior reports have shown inconsistent associations and disagreements among symptoms, signs, and clinical findings in patients with dry eye disease and MGD. ${ }^{29-32}$ Because patients received laser treatment once per month over four treatments, a longer treatment period may have been needed to 
maintain effectiveness; this longer period might significantly improve symptoms and signs of dry eye disease and other clinical parameters that are related to MGD.

Combined application of 2940-nm Er:YAG and 1064-nm Nd:YAG lasers had an acceptable safety profile. All adverse events were mild and resolved within 1 week without any treatment. The most common adverse event was conjunctival hyperaemia, which resolved spontaneously within 1 day. The use of a corneal metal shield during intervention was presumed to be the cause of short-term conjunctival hyperemia. Uncorrected visual acuity, best-corrected visual acuity, and intraocular pressure did not change during this study.

There were some potential limitations in this study. First, this was an observational cohort study; further randomised controlled trials comparing this combination laser treatment and placebo are needed to confirm the effectiveness of this approach. Second, patients were only treated four times, then evaluated at 16 and 24 weeks after baseline. Although this study showed effectiveness in terms of meibum quality score improvement, greater treatment frequency and longer evaluation are needed to validate the long-term effects of the laser treatment protocol. Lastly, additional evidence regarding inflammatory biological markers (eg, tear cytokines involved in MGD) is needed to ascertain the biological effects and evaluate adverse effects (eg, ocular inflammation) of this laser treatment.

\section{Conclusions}

Combined application of three modes of 2940-nm Er:YAG and 1064-nm Yd:YAG lasers significantly improved meibum quality in patients with MGD and ameliorated the symptoms and signs of dry eye disease. Its effectiveness was limited to approximately 4 weeks after completion of laser treatment; the effects were absent at 12 weeks after completion of laser treatment.

\section{Acknowledgments}

We thank the Chula Refractive Surgery Center, Department of Ophthalmology, King Chulalongkorn Memorial Hospital, Thailand for providing a study site and study personnel. Moreover, we would like to thank all patients in this study.

\section{Disclosure}

The authors report no conflicts of interest in this work.

\section{References}

1. Knop E, Knop N, Millar T, Obata H, Sullivan DA. The international workshop on meibomian gland dysfunction: report of the subcommittee on anatomy, physiology, and pathophysiology of the meibomian gland. Invest Ophthalmol Vis Sci. 2011;52(4):1938-1978. doi:10.1167/iovs.10-6997c

2. Geerling G, Tauber J, Baudouin C, et al. The international workshop on meibomian gland dysfunction: report of the subcommittee on management and treatment of meibomian gland dysfunction. Invest Ophthalmol Vis Sci. 2011;52(4):2050-2064. doi:10.1167/iovs.10$6997 \mathrm{~g}$

3. Stevenson W, Chauhan SK, Dana R. Dry eye disease: an immune-mediated ocular surface disorder. Arch Ophthalmol. 2012;130(1):90-100. doi:10.1001/archophthalmol.2011.364

4. Lam PY, Shih KC, Fong PY, et al. A review on evidence-based treatments for meibomian gland dysfunction. Eye Contact Lens. 2020;46(1):3-16. doi:10.1097/ICL.0000000000000680

5. Ferrari G, Colucci A, Barbariga M, Ruggeri A, Rama P. High frequency electrotherapy for the treatment of meibomian gland dysfunction. Cornea. 2019;38(11):1424-1429. doi:10.1097/ICO.000 0000000002063

6. Toyos R, McGill W, Briscoe D. Intense pulsed light treatment for dry eye disease due to meibomian gland dysfunction; a 3-year retrospective study. Photomed Laser Surg. 2015;33(1):41-46. doi:10.1089/pho.2014.3819

7. Pedrotti E, Bosello F, Fasolo A, et al. Transcutaneous periorbital electrical stimulation in the treatment of dry eye. Br J Ophthalmol. 2017;101(6):814-819. doi:10.1136/bjophthalmol-2016-308678

8. Gaspar A, Brandi H, Gomez V, Luque D. Efficacy of Erbium: YAG laser treatment compared to topical estriol treatment for symptoms of genitourinary syndrome of menopause. Lasers Surg Med. 2017;49 (2):160-168. doi:10.1002/1sm.22569

9. Drnovsek-Olup B, Beltram M, Pizem J. Repetitive Er:YAG laser irradiation of human skin: a histological evaluation. Lasers Surg Med. 2004;35(2):146-151. doi:10.1002/lsm.20080

10. Kunzi-Rapp K, Dierickx CC, Cambier B, Drosner M. Minimally invasive skin rejuvenation with Erbium: YAG laser used in thermal mode. Lasers Surg Med. 2006;38(10):899-907. doi:10.1002/ $1 \mathrm{sm} .20380$

11. Karabudak O, Dogan B, Baloglu H. Histologic evidence of new collagen formation using a Q-switched Nd: YAGlaser in periorbital rhytids. J Dermatolog Treat. 2008;19(5):300-304. doi:10.1080/ 09546630801961075

12. Fistonić N, Fistonić I, Guštek ŠF, et al. Minimally invasive, non-ablative Er: YAGlaser treatment of stress urinary incontinence in women--a pilot study. Lasers Med Sci. 2016;31(4):635-643. doi:10.1007/s10103-016-1884-0

13. Bodendorf MO, Willenberg A, Anderegg U, Grunewald S, Simon JC, Paasch U. Connective tissue response to fractionated thermo-ablative Erbium: YAG skin laser treatment. Int J Cosmet Sci. 2010;32 (6):435-445. doi:10.1111/j.1468-2494.2009.00567.x

14. Clark RA. Cutaneous tissue repair: basic biologic considerations. I. J Am Acad Dermatol. 1985;13(5 Pt 1):701-725. doi:10.1016/S01909622(85)70213-7

15. Butovich IA, Millar TJ, Ham BM. Understanding and analyzing meibomian lipids--a review. Curr Eye Res. 2008;33(5):405-420. doi:10.1080/02713680802018419

16. Dell SJ. Intense pulsed light for evaporative dry eye disease. Clin Ophthalmol. 2017;11:1167-1173. doi:10.2147/OPTH.S139894

17. Nichols KK, Foulks GN, Bron AJ, et al. The international workshop on meibomian gland dysfunction: executive summary. Invest Ophthalmol Vis Sci. 2011;52(4):1922-1929. doi:10.1167/iovs.106997a 
18. Miller KL, Walt JG, Mink DR, et al. Minimal clinically important difference for the ocular surface disease index. Arch Ophthalmol. 2010;128(1):94-101. doi:10.1001/archophthalmol.2009.356

19. Bron AJ, Evans VE, Smith JA. Grading of corneal and conjunctival staining in the context of other dry eye tests. Cornea. 2003;22 (7):640-650. doi:10.1097/00003226-200310000-00008

20. Tomlinson A, Bron AJ, Korb DR, et al. The international workshop on meibomian gland dysfunction: report of the diagnosis subcommittee. Invest Ophthalmol Vis Sci. 2011;52(4):2006-2049. doi:10.1167/iovs.10-6997f

21. Terry RL, Schnider CM, Holden BA, et al. CCLRU standards for success of daily and extended wear contact lenses. Optom Vis Sci. 1993;70(3):234-243. doi:10.1097/00006324-199303000-00011

22. Arita R, Shirakawa R, Maeda S, Yamaguchi M, Ohashi Y, Amano S. Decreased surface temperature of tarsal conjunctiva in patients with meibomian gland dysfunction. JAMA Ophthalmol. 2013;131 (6):818-819. doi:10.1001/jamaophthalmol.2013.1895

23. Zhou S, Robertson DM. Wide-field in vivo confocal microscopy of meibomian gland acini and rete ridges in the eyelid margin. Invest Ophthalmol Vis Sci. 2018;59(10):4249-4257. doi:10.1167/iovs.1824497

24. Majaron B, Srinivas SM, Huang H, Nelson JS. Deep coagulation of dermal collagen with repetitive Er: YAGlaser irradiation. Lasers Surg Med. 2000;26(2):215-222. doi:10.1002/(SICI)1096-9101(2000) 26:2<215::AID-LSM13>3.0.CO;2-O

25. Dams SD, de Liefde-van Beest M, Nuijs AM, Oomens CW, Baaijens FP. Heat shocks enhance procollagen type I and III expression in fibroblasts in ex vivo human skin. Skin Res Technol. 2011;17 (2):167-180. doi:10.1111/j.1600-0846.2010.00473.x
26. Pourzarandian A, Watanabe H, Ruwanpura SM, Aoki A, Noguchi K, Ishikawa I. Er:YAG laser irradiation increases prostaglandin E2 production via the induction of cyclooxygenase- 2 mRNA in human gingival fibroblasts. $J$ Periodontal Res. 2005;40(2):182-186. doi:10.1111/j.1600-0765.2005.00789.x

27. Hausman GJ, Richardson RL. Cellular and vascular development in immature rat adipose tissue. J Lipid Res. 1983;24(5):522-532. doi:10.1016/S0022-2275(20)37957-8

28. Lee JH, Park SR, Jo JH, Park SY, Seo YK, Kim SM. Comparison of epidermal/dermal damage between the long-pulsed $1064 \mathrm{~nm} \mathrm{Nd:}$ YAGand $755 \mathrm{~nm}$ alexandrite lasers under relatively high fluence conditions: quantitative and histological assessments. Photomed Laser Surg. 2014;32(7):386-393. doi:10.1089/pho.2013.3665

29. Cuevas M, González-García MJ, Castellanos E, et al. Correlations among symptoms, signs, and clinical tests in evaporative-type dry eye disease caused by Meibomian gland dysfunction (MGD). Curr Eye Res. 2012;37(10):855-863. doi:10.3109/02713683.2012.683508

30. Bartlett JD, Keith MS, Sudharshan L, Snedecor SJ. Associations between signs and symptoms of dry eye disease: a systematic review. Clin Ophthalmol. 2015;9:1719-1730. doi:10.2147/OPTH. S89700

31. Onwubiko SN, Eze BI, Udeh NN, Onwasigwe EN, Umeh RE. Dry eye disease: concordance between the diagnostic tests in African eyes. Eye Contact Lens. 2016;42(6):395-400. doi:10.1097/ ICL.0000000000000218

32. Kyei S, Dzasimatu SK, Asiedu K, Ayerakwah PA. Association between dry eye symptoms and signs. J Curr Ophthalmol. 2018;30 (4):321-325. doi:10.1016/j.joco.2018.05.002
Clinical Ophthalmology

\section{Publish your work in this journal}

Clinical Ophthalmology is an international, peer-reviewed journal covering all subspecialties within ophthalmology. Key topics include: Optometry; Visual science; Pharmacology and drug therapy in eye diseases; Basic Sciences; Primary and Secondary eye care; Patient Safety and Quality of Care Improvements. This journal is indexed on PubMed
Dovepress

Central and CAS, and is the official journal of The Society of Clinical Ophthalmology (SCO). The manuscript management system is completely online and includes a very quick and fair peer-review system, which is all easy to use. Visit http://www.dovepress.com/ testimonials.php to read real quotes from published authors. 